\title{
Untangling the evolution of heavy ion collisions using direct photon interferometry
}

\author{
Oscar Garcia-Montero, ${ }^{a, *}$ Nicole Löher, ${ }^{b}$ Aleksas Mazeliauskas, ${ }^{c}$ Klaus Reygers ${ }^{b}$ \\ and Jürgen Berges ${ }^{d}$ \\ a Institute for Theoretical Physics, Goethe University \\ Max-von-Laue-Strasse 1, 60438 Frankfurt am Main, Germany \\ ${ }^{b}$ Physikalisches Institut, Universität Heidelberg, \\ Im Neuenheimer Feld 226, 69120 Heidelberg, Germany \\ ${ }^{c}$ Theoretical Physics Department, CERN, \\ CH-1211 Genève 23, Switzerland \\ ${ }^{d}$ Institut für Theoretische Physik, Universität Heidelberg \\ Philosophenweg 16, 69120 Heidelberg, Germany.
}

E-mail: garcia@fias.uni-frankfurt.de

Recently, two scenarios have been proposed to resolve the discrepancy between photon yield and the flow coefficients measured in nuclear collisions at RHIC and the LHC. In the first, additional photons are produced from the early pre-equilibrium stage computed from the "bottom-up" thermalization scenario. In the second, the thermal rates are enhanced close to the pseudo-critical temperature $T_{c} \sim 155 \mathrm{MeV}$ using a phenomenological ansatz. We investigate the measurement of Hanbury Brown-Twiss (HBT) photon correlations as an experimental tool to discriminate between such scenarios. By enhancing standard hydrodynamical simulations with these scenarios, we compute the correlators in terms of the relative momenta of the pair for different values of its transverse momenta, $K_{\perp}$. We find that the longitudinal correlation is the most sensitive to different photon sources.

HardProbes 2020

1-6 June 2020

Austin, Texas

${ }^{*}$ Speaker 


\section{Introduction}

Electromagnetic probes produced during Heavy Ion Collisions (HIC), and particularly direct photons, which are photons which are not produced in hadronic decay, are often quoted to be excellent probes to explore the space-time evolution of the fireball. This is because, as they do not present strong interactions with the medium, they escape from the medium to the detector virtually unscathed. However, they are produced continuously during the evolution of the medium, and because the final measurement cannot present time-resolution, discrimination between different sources and their importance throughout the collision is extremely difficult. To do this, one needs access to higher correlations, which in the case of photons, will be more sensitive to the evolution of the system than particle spectra. In this work we put forward photon Hanbury-Brown-Twiss (HBT) correlations[1], which are normalized 2-photon correlations, as a tool that can be used both experimentally and theoretically to start to separate the different sources. To showcase how one can do that, two possible sources for enhancing the invariant photon yield are discussed. The first is a pre-thermal source, based on the first stage of the bottom-up thermalization scenario [2,3]. The second one, is an phenomenological model of enhancement for the thermal rates near a pseudocritical temperature, $T_{p c}$, see Ref. [4]. We will show how the inclusion of additional sources can affect the correlators, and how their change can be used to discriminate different models of photon production.

\section{Sources of Photon Production}

In this work, we produce direct photons for $\mathrm{Pb}-\mathrm{Pb}$ at $\sqrt{s_{\mathrm{NN}}}=2.76 \mathrm{TeV}$, in the $0-20 \%$ centrality class. We will separate the results into photons which are produced in our base calculation, and those which are produced by adding either the pre-equilibrium stage, or a phenomenological enhancement, as it was stated in the introduction. The former contains the contribution of prompt photons, hard probes produced at initial time, as well as thermal photons from the quark-gluon-plasma (QGP) and hadronic stages. Prompt photons were produced perturbatively from partonic scattering from binary nucleon-nucleon collisions [5], and scaled up using the Glauber model. On the other hand, thermal photons were calculated by folding thermal rates [6,7] with a $2+1 \mathrm{D}$ hydrodynamical event-by-event simulation. The evolution of the hydrodynamical medium was performed using the VISHNU package [8], starting at $\tau_{\text {hydro }}=0.6 \mathrm{fm}$. Full details on our simulation can be found in Ref. [9]. Apart from our base calculation, we have included two separate scenarios for early and late-time enhancement, which will be summarized in what follows.

\subsection{Photon production from pre-equilibrium}

Recent estimates have shown that photon production from the non-equilibrium stages prior to thermalization is non-negligible compared the total direct-photon yield [2]. To account for a source of pre-equilibrium photons we use the bottom-up scenario [3], where gluon saturation is assumed to take place at RHIC and LHC energies. The evolution is then described by strong, non-linear macroscopic fields, which rapidly increase gluon occupation. This highly occupated state relaxes via turbulent thermalization [10]. After this, low-energy modes grow in number, creating a "soft bath", and thermalization is achieved after all highly energetic gluons loose their energy to the soft bath. We will evolve the pre-equilibrium stage from $\tau_{0} \sim Q_{s}^{-1}$, where $Q_{s}$ is the saturation scale of 

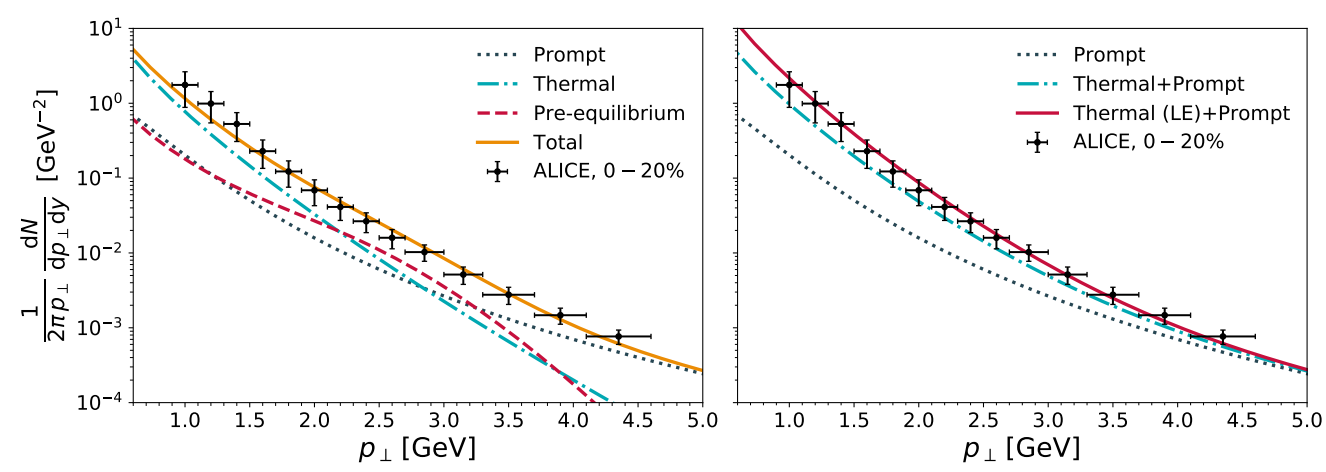

Figure 1: Comparison of the $p_{\perp}$ spectra of direct photons from different sources to ALICE measurement in central $(0-20 \%) \mathrm{Pb}-\mathrm{Pb}$ collisions at $\sqrt{s_{\mathrm{NN}}}=2.76 \mathrm{TeV}$ [11]. Left: contributions from prompt (dotted line), thermal (dash-dotted line) and pre-equilibrium (dashed line) sources. The total result (sum of prompt, thermal and pre-equilibrium photons) is shown by the solid line. Right: one can see the combined prompt and thermal photon spectrum (dash-dotted line) with the late-time enhancement (LE) scenario (solid line).

the system, up to $\tau_{\text {hydro }}$, where we perform a matching of the energy density to get the saturation scale. The initial time is then set using the average $Q_{s}$. Photon production in this setting is done with a small-angle approximated kinetic rate,

$$
E \frac{\mathrm{d} N}{\mathrm{~d}^{4} x \mathrm{~d}^{3} p}=\frac{10}{9 \pi^{4}} \alpha \mathcal{L} Q_{s}^{2} \kappa_{g}\left(\frac{\tau_{0}}{\tau}\right) f_{q}(\tau, \mathbf{p}),
$$

where $\alpha$ is the electromagnetic coupling, $\kappa_{g}=c\left(2 N_{c}\right)^{-1}$, where $N_{c}$ is the number of colors, and $c$ is the gluon liberation factor described in Ref. [12]. The quark distribution, $f_{q}$ is assumed to inherit the scaling properties of the parent gluon distribution, taken from classical-statistical simulations. To do this, we use $f_{q} \sim \alpha_{S} f_{g}$, and account form spin statistics. ${ }^{1}$

\subsection{Critical enhancement at late times near $T_{c}$}

We will use a late-time source to contrast the early-time production. We chose the phenomenological model of enhancement from Ref. [4]. There, photon rates are enhanced around a pseudo-critical temperature, $T_{c}$, close to the transition to hadronic degrees of freedom, since partonic cross-sections are expected to rise fast, as a result of confining interactions. For the purpose of this work, we model the enhancement factor, $h(T)$, as

$$
E \frac{d N_{\mathrm{enh}}}{\mathrm{d}^{4} x \mathrm{~d}^{3} p} \equiv h(T) E \frac{d N_{\text {thermal }}}{\mathrm{d}^{4} x \mathrm{~d}^{3} p}, \quad \text { with } \quad h(T)=1+h_{0} \exp \left\{-\frac{\left(T-T_{\mathrm{c}}\right)^{2}}{d^{2}}\right\} .
$$

We have set the enhancement parameters here to be $T_{\mathrm{c}}=155 \mathrm{MeV}, h_{0}=3$ and $d=50 \mathrm{MeV}$. The latter two were chosen to match the experimental results from ALICE (see Fig. 1).

\section{Results}

The full computation of direct photons for the base calculation, as well as for the added sources can be found in Fig. 1. One can see that good agreement with experimental data can be found

\footnotetext{
${ }^{1}$ The fully detailed account of this computation can be found in Ref. [9] while a calculation of photon production of the complete 'bottom-up' can be found in Ref. [13].
} 


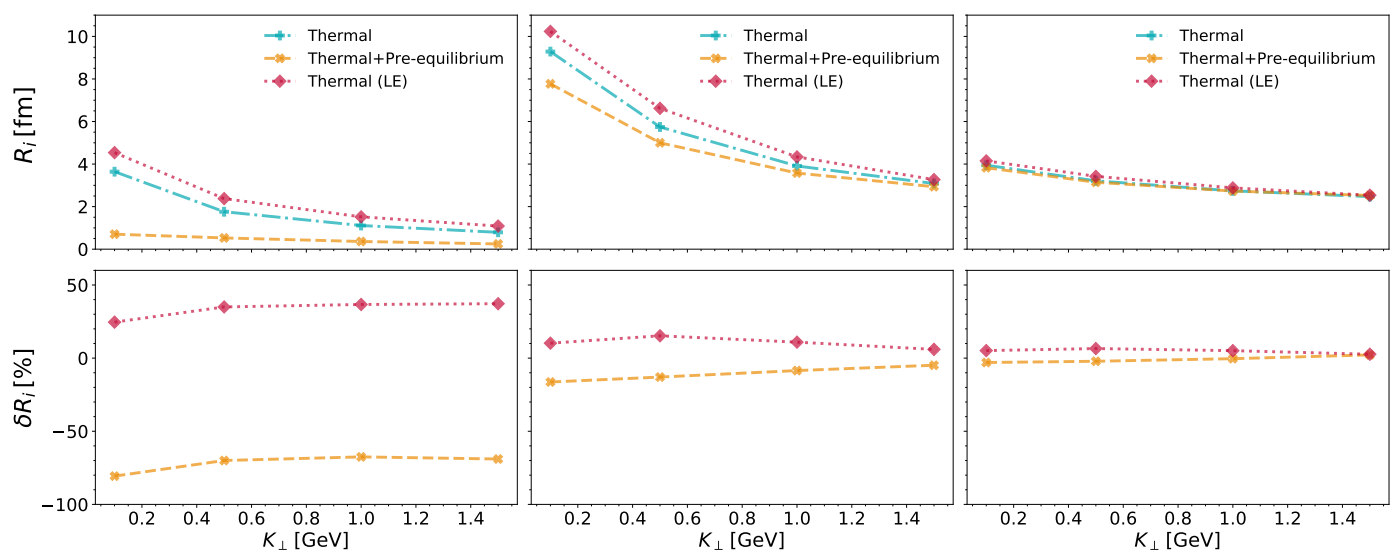

Figure 2: Left: HBT radii as a function of the pair momenta, calculated using the method of moments. Right: Percentage change of the radii for the two scenarios.

both for the pre-equilibrium case, as well as for the late-time enhancement. For this reason and to then showcase HBT as a potential tool for the discrimination of sources, we computed the 2-photon distributions by folding the rates cited above with their respective space-time evolution. From them, we calculated the photon HBT correlation,

$$
C(\boldsymbol{q}, \boldsymbol{K})=\left[E_{\boldsymbol{p}_{1}} E_{\boldsymbol{p}_{2}} \frac{\mathrm{d} N}{\mathrm{~d}^{3} p_{1} \mathrm{~d}^{3} p_{2}}\right] /\left[E_{\boldsymbol{p}_{1}} \frac{\mathrm{d} N}{\mathrm{~d}^{3} p_{1}} E_{\boldsymbol{p}_{2}} \frac{\mathrm{d} N}{\mathrm{~d}^{3} p_{2}}\right] .
$$

where we denote $q=p_{1}-p_{2}$ and $K=\left(p_{1}+p_{2}\right) / 2$, the relative and average momentum, respectively. It is useful to think in terms of projection variables, that is a coordinate system in which we align the $z$ axis with the beam, and the $x$-axis with the direction of the average momentum of the photons, $K^{\mu}=\left(K^{0}, K_{\perp}, 0, K^{z}\right)$ and $q^{\mu}=\left(q^{0}, q_{\mathrm{o}}, q_{\mathrm{s}}, q_{1}\right)$. Here, $o, s, l$ stand for outwards, sidewards and longitudinal, respectively. The HBT correlator, Eq. (3), was computed for midrapidity pairs, $K_{z}=0$, along the three diagonals, i.e. $q_{i}$ with $q_{j}=q_{k}=0$ for $i \neq j \neq k$. The longitudinal correlators were the most sensitive to the addition of extra sources, as expected. The effect can be better seen in the HBT radii, which can be found Fig. 2. We extracted the radii from the aforementioned correlators using the characteristic scale method. Just as the correlators, the longitudinal radii are the most sensitive to the sources, where they changed between $20-80 \%$, while the outwards and sidewards radii exhibit no more than $20 \%$ change. In Ref. [9] the reader can find a more detailed discussion of these results, as well as other useful observables.

\section{Summary}

We present this study as a showcase for HBT to be used as a tool for discrimination of known and novel photon sources in the evolution of HICs. We propose to use the correlators, and the corresponding radii to as an extra experimental and theoretical observable to further test scenarios of photon production. To do this, we have used to distinct sources, one in which additional photons are produced from a pre-thermal evolution, and one in which the thermal stages have enhanced photon production close to the pseudo-critical taemperature. We find that both cases agree with experimental data for the invariant yields. We use these two settings to compute the HBT correlator, 
and with it, the HBT radii. We find substantial, and opposed, change in these, particularly in the longitudinal direction, and such change may be used to understand the importance of the sources of photon production.

\section{References}

[1] E. Frodermann and U. Heinz, Photon HBT interferometry for non-central heavy-ion collisions, Phys. Rev. C80 (2009) 044903

[2] J.Berges, K. Reygers, N. Tanji and R. Venugopalan, Parametric estimate of the relative photon yields from the glasma and the quark-gluon plasma in heavy-ion collisions, Phys. Rev. C95 (2017) 5, 054904"

[3] R. Baier, A.H. Mueller, D. Schiff and D.T. Son, 'Bottom up' thermalization in heavy ion collisions Phys. Lett. B502 (2001) 51-58, 024915

[4] H. van Hees, M. He and R. Rapp, Pseudo-critical enhancement of thermal photons in relativistic heavy-ion collisions?. Nucl. Phys. A933 (2015) 256-271

[5] L.E. Gordon and W. Vogelsang, Polarized and unpolarized prompt photon production beyond the leading order, Phys. Rev. D48 (1993) 3136-3159

[6] P. B. Arnold , G.D. Moore and L.G. Yaffe, Photon emission from quark gluon plasma: Complete leading order results. JHEP12 (2001) 009

[7] M. Heffernan, P. Hohler and R. Rapp, Universal Parametrization of Thermal Photon Rates in Hadronic Matter Phys. Rev. C91 (2015) 2, 027902

[8] C.Shen, Z. Qiu, H. Song, J. Bernard, S. Bass and U. Heinz, The iEBE-VISHNU code package for relativistic heavy-ion collisions, Comp. Phys. Comm. 199 (2016) 61 - 85

[9] O. Garcia-Montero, N. Löher, A. Mazeliauskas, J. Berges and K. Reygers, Probing the evolution of heavy-ion collisions using direct photon interferometry,. Phys. Rev. C102 (2020) 2, 024915

[10] J.Berges, K. Boguslavski, S. Schlichting and R. Venugopalan, Turbulent thermalization process in heavy-ion collisions at ultrarelativistic energies", Phys. Rev. D89 (2014) 7, 074011

[11] J. Adam et al, Direct photon production in $\mathrm{Pb}-\mathrm{Pb}$ collisions at $\mathrm{sNN}=2.76 \mathrm{TeV}$. Physics Letters B754 (2016), 235 - 248

[12] T. Lappi, Wilson line correlator in the MV model: Relating the glasma to deep inelastic scattering, Eur. Phys. J. C55 (2008) 285-292

[13] O. Garcia-Montero, Non-equilibrium photons from the bottom-up thermalization scenario. [arXiv: 1909.12294]. 